\title{
Pseudo-insular glioma syndrome: illustrative cases
}

\author{
Alexander F. Haddad, MD, ${ }^{1}$ Jacob S. Young, MD, ${ }^{1}$ Ramin A. Morshed, MD, ${ }^{1}$ S. Andrew Josephson, MD, ${ }^{2}$ Soonmee Cha, MD, ${ }^{3}$ and \\ Mitchel S. Berger, MD ${ }^{1}$
}

Departments of ${ }^{1}$ Neurological Surgery, ${ }^{2}$ Neurology, and ${ }^{3}$ Radiology, University of California, San Francisco, San Francisco, California

BACKGROUND Lower-grade insular gliomas often appear as expansile and infiltrative masses on magnetic resonance imaging (MRI). However, there are nonneoplastic lesions of the insula, such as demyelinating disease and vasculopathies, that can mimic insular gliomas.

OBSERVATIONS The authors report two patients who presented with headaches and were found to have mass lesions concerning for lower-grade insular glioma based on MRI obtained at initial presentation. However, on the immediate preoperative MRI obtained a few weeks later, both patients had spontaneous and complete resolution of the insular lesions.

LESSONS Tumor mimics should always be in the differential diagnosis of brain masses, including those involving the insula. The immediate preoperative MRI (within 24-48 hours of surgery) must be compared carefully with the initial presentation MRI to assess interval change that suggests tumor mimics to avoid unnecessary surgical intervention.

https://thejns.org/doi/abs/10.3171/CASE21481

KEYWORDS low-grade glioma; vasculopathy; mimic; MRl; imaging

Lower-grade gliomas (LGGs) are slow-growing glial tumors with the potential for malignant transformation. The incidence is highest in patients in their third or fourth decade of life, and both the tumor and the treatments can significantly impact patients' quality of life. Seizure is the most common presenting symptom in the majority of patients, but headache and focal neurological deficits are also frequently seen. ${ }^{1,2}$ Indeed, LGGs are more often present in highly functional areas of the brain, with the insula and supplemental motor area being the two most commonly afflicted locations. ${ }^{3,4}$ On magnetic resonance imaging (MRI), insular gliomas appear as expansile and infiltrative masses that are hyperintense on fluid-attenuated inversion recovery (FLAIR) sequences and hypointense on T1-weighted precontrast sequences, and they show no enhancement on T1-weighted postcontrast sequences. ${ }^{5-7}$ On proton magnetic resonance (MR) spectroscopy, LGGs will frequently demonstrate an increased choline peak and a decreased $\mathrm{N}$-acetyl aspartate peak. ${ }^{5-7}$ The standard of care for LGGs includes surgical intervention, with the goal of providing maximal safe resection and acquisition of adequate tissue for detailed molecular and genetic characterization. ${ }^{8}$ This is becoming increasingly important as the molecular subtypes of LGGs and their unique characteristics are becoming increasingly well defined. ${ }^{9}$

Despite the characteristic clinical symptoms and imaging findings associated with LGGs, it is critical to maintain a broad differential diagnosis when assessing these patients, including nonneoplastic conditions that can act as tumor mimics, such as demyelinating diseases, autoimmune conditions (e.g., neurosarcoid), other neoplasms, atypical infections, and vasculopathies. Preoperative MRI in close proximity to a planned resection (i.e., within 24-48 hours) should also be obtained to allow an additional assessment of the lesion before surgical intervention and to assess for any interval change that may suggest a resolving nonneoplastic process. We report cases of two patients who presented with symptoms and imaging findings concerning for LGG of the insula and in both of whom there was spontaneous resolution of the insular lesion on the immediate preoperative MRI.

ABBREVIATIONS ANA = antinuclear antibodies; CNS = central nervous system; $C S F=$ cerebrospinal fluid CT $=$ computed tomography; DTI = diffusion tensor imaging; $D W I$ = diffusion-weighted imaging; FLAIR = fluid-attenuated inversion recovery; $L G G=$ lower-grade glioma; $M C A=$ middle cerebral artery; $M R=$ magnetic resonance; $\mathrm{MRI}=$ magnetic resonance imaging; $M S$ = multiple sclerosis; PACNS = primary angiitis of the central nervous system; $P E T=$ positron emission tomography; $\mathrm{PWI}=$ perfusion-weighted imaging; $\mathrm{TDL}=$ tumefactive demyelinating lesion.

INCLUDE WHEN CITING Published December 27, 2021; DOI: 10.3171/CASE21481.

SUBMITTED August 24, 2021. ACCEPTED September 20, 2021.

(C) 2021 The authors, CC BY-NC-ND 4.0 (http://creativecommons.org/licenses/by-nc-nd/4.0/). 


\section{Illustrative Cases \\ Case 1}

A 37-year-old right-handed woman presented with new-onset left-sided headaches with outside hospital imaging concerning for a new left frontotemporal brain mass. She was neurologically intact as assessed using telehealth modalities. T2 and T2 FLAIR MRI scans of the brain revealed a focal area of hyperintensity in zone IV of the left insula (Fig. 1A-C). Postcontrast T1-weighted imaging also revealed a corresponding hypointense area in zone IV of the insula, with perivascular enhancement around branches of the left middle cerebral artery (MCA) (Fig. 1D-F). The imaging findings were most consistent with an LGG. As a result, repeat imaging studies were scheduled prior to potential surgical intervention with the goal of a maximal safe resection and obtaining tissue for molecular phenotyping of the potential tumor. These repeat imaging studies approximately 7 weeks following initial imaging revealed resolution of the zone IV insular lesion on T2 and T2 FLAIR imaging, as well as T1 postcontrast sequences (Fig. 2A-F). Interval imaging also showed resolution of the T1 postcontrast enhancement around the left MCA branches. Of note, steroids were not initiated at any time prior to repeat imaging. As a result of this imaging resolution, surgical intervention was not performed, and the patient was instead scheduled for repeat MRI of the brain. Testing of the patient's cerebrospinal fluid (CSF) revealed pleocytosis with 6 white blood cells per cubic millimeter (83\% lymphocytes, $8 \%$ neutrophils, and $9 \%$ monocytes/macrophages) and two unique oligoclonal bands that were not present in a corresponding serum sample. The result of Gram staining of the CSF was negative for microorganisms. Routine serum autoimmune markers, including antinuclear antibodies (ANA) and Sjögren's antibodies, were unremarkable.

\section{Case 2}

A 41-year-old right-handed woman with a history of migraines presented to our clinic with a change in the quality of her headaches. She was neurologically intact as assessed using telehealth modalities. In a similar manner to the previous case, T2 MRI of the brain showed a focal area of hyperintensity in zone IV of the left insula (Fig. 3A and B). This lesion was hypointense on postcontrast T1-weighted imaging (Fig. 3C and D). T1 postcontrast imaging also demonstrated perivascular enhancement around branches of the left MCA (Fig. $3 C$ and D). Similar to case 1 , this patient was scheduled for surgical resection with a preoperative diagnosis of LGG of the insula. However, repeat MRI performed 6 weeks later showed resolution of both the left MCA enhancement and the insular lesion (Fig. 4A-D). Resolution of the lesion was stable on repeat imaging 11 months following the initial MRI of the patient. No steroids had been initiated prior to resolution of the lesion, and CSF studies were not obtained.

\section{Discussion}

\section{Observations}

In this report, we highlight the cases of two patients with imaging findings initially concerning for LGG of the insula, but with interval
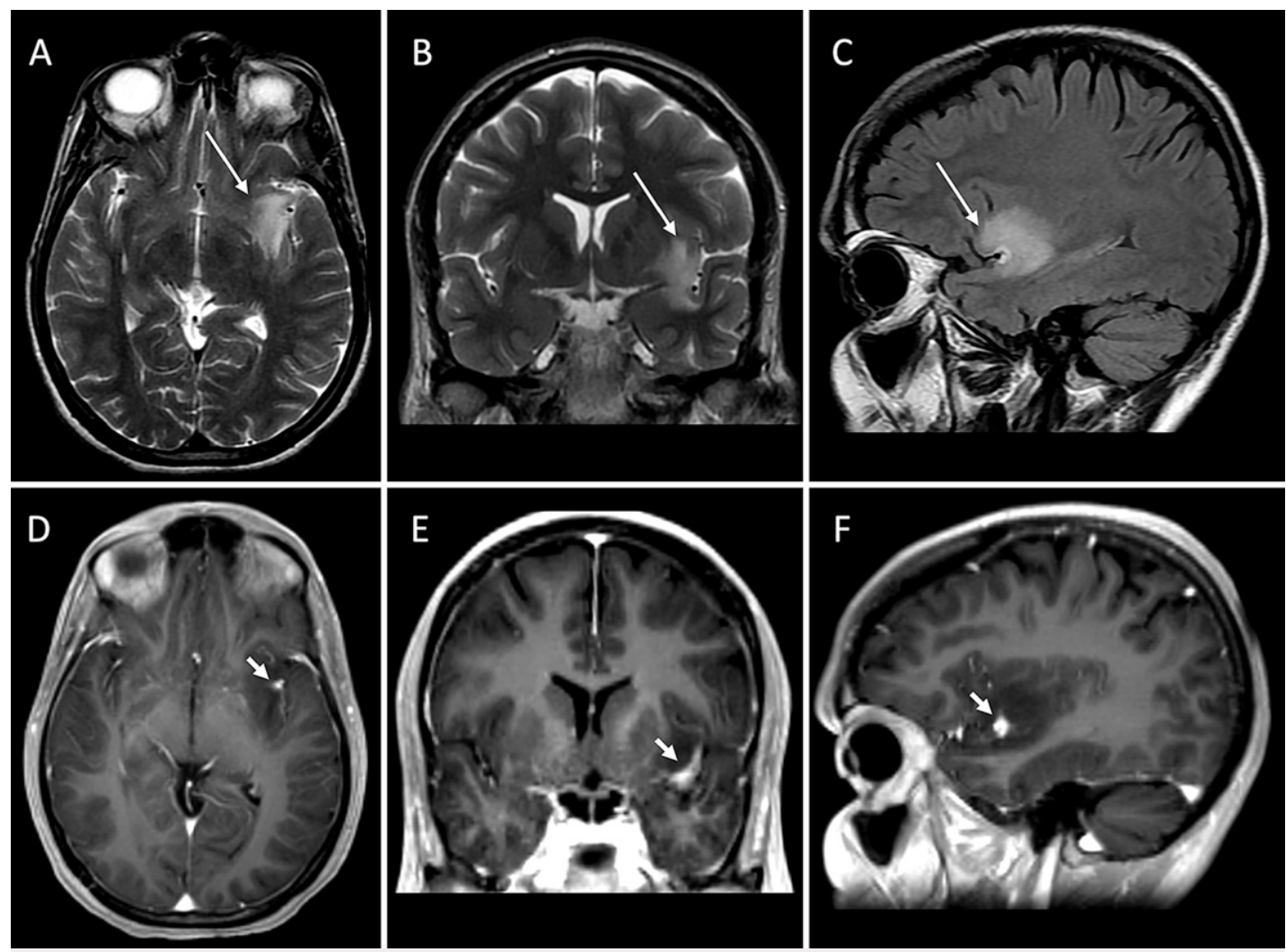

FIG. 1. Case 1 at initial presentation. A-C: Axial T2-weighted (A), coronal T2-weighted (B), and sagittal FLAIR (C) images show expansile mass lesion in the left insula (long arrows). D-F: Axial (D), coronal (E), and sagittal (F) postcontrast T1weighted images show discrete area of abnormal enhancement (short arrows) in the region of the left MCA. 

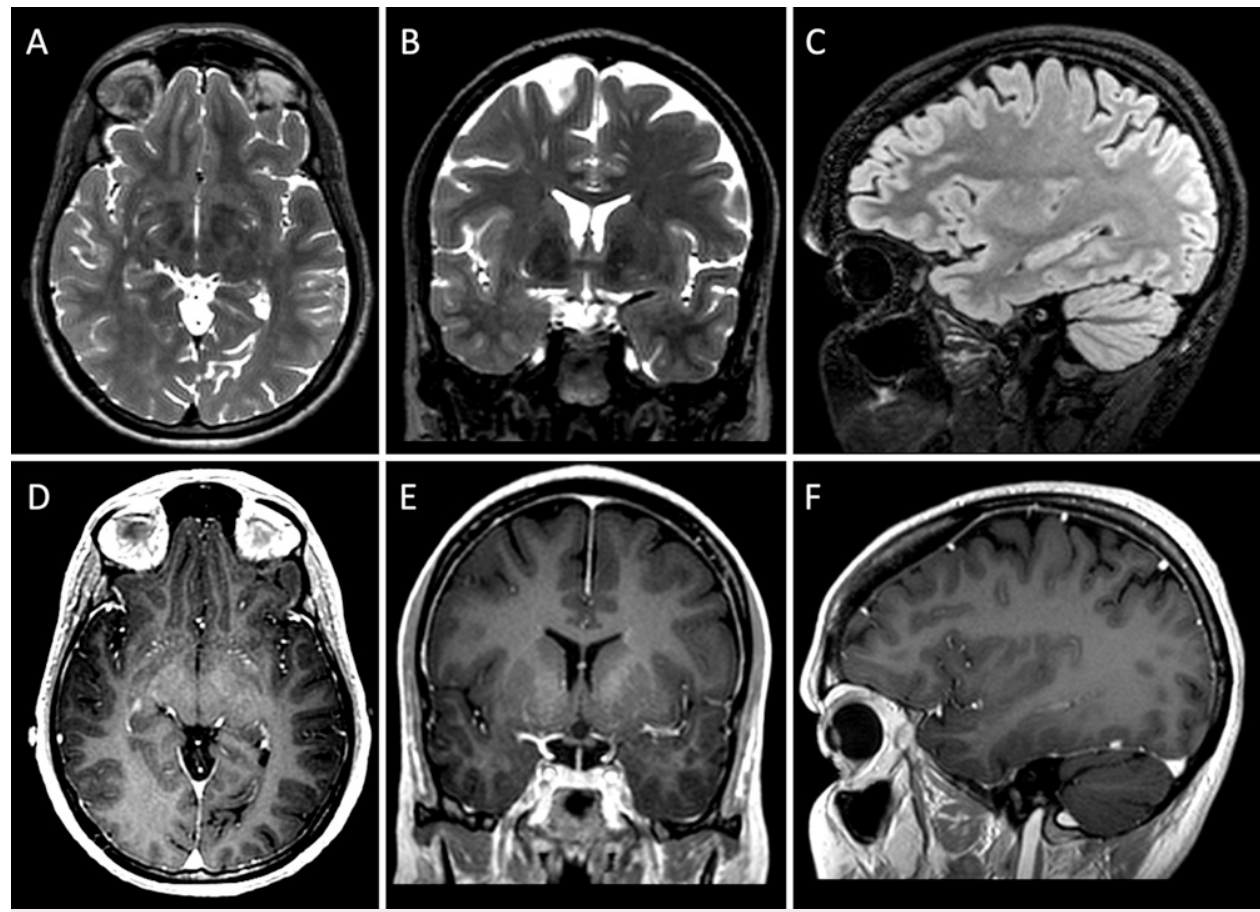

FIG. 2. Case 1 at preoperative MRI. A-C: Axial T2-weighted (A), coronal T2-weighted (B), and sagittal FLAIR (C) images show complete resolution of expansile mass lesion in the left insula. D-F: Axial (D), coronal (E), and sagittal (F) postcontrast T1-weighted images show no residual enhancement in the region of left MCA.

resolution of their lesions on repeat preoperative imaging. Interestingly, both patients were female, presented with headache, were between 35 and 45 years of age, and demonstrated asymmetrical perivascular enhancement of MCA branches on T1-weighted postcontrast imaging ipsilateral to the lesions. These cases demonstrate the importance of considering LGG mimics prior to surgical intervention and the utility of obtaining repeat imaging in the immediate preoperative time period, within 24 hours of surgery, both for stereotactic navigation use and to evaluate any changes in a tumor prior to intervention.

MRI is critical for LGG diagnosis and can be quite accurate, with positive and negative predictive values ranging from $79 \%$ to $92 \%$ and $44 \%$ to $86 \%$, respectively. ${ }^{10-12}$ These values improve with additional sequences such as perfusion-weighted imaging (PWI), diffusion-weighted imaging (DWI), and proton MR spectroscopy. ${ }^{13}$ Positron emission tomography (PET) has also been shown to have high sensitivity and specificity, ranging from $93 \%$ to $100 \%$ and $67 \%$ to $100 \%$, respectively, in the diagnosis of $L G G$ and in distinguishing LGG from high-grade glioma. ${ }^{14,15}$ Interestingly, MRI has also shown some ability to differentiate between molecular subtypes of LGGs, an exciting development that will almost certainly continue to grow as imaging and machine learning techniques improve. ${ }^{16,17} \mathrm{MRI}$ also plays a critical role in the surgical planning of LGGs. A repeat preoperative MRI provides surgeons with a more accurate localization of the tumor and its characteristics, which is important for surgical planning. Our practice is to obtain a high-quality in-house MRI scan 24-48 hours prior to surgery with diffusion tensor imaging (DTI) and fiducials. DTI allows the visualization of white matter tracts, with improved ability to perform maximal safe resection. ${ }^{18,19}$ Indeed, a randomized clinical trial of 238 patients evaluating the utility of
DTI by Wu et al. demonstrated improved postoperative Karnofsky scores in patients with LGG who had undergone resection and had DTI information available to surgeons, highlighting the utility of this technique. ${ }^{20}$ Finally, MRI also serves as a means of postoperative surveillance, with serial T2 FLAIR and T1-weighted pre- and postcontrast imaging frequently used and increasing contrast enhancement associated with progression or transformation into a highergrade glioma. $^{13}$

Nevertheless, although MRI plays an important part in the diagnosis and characterization of LGGs, surgeons should also consider potential brain tumor mimics. Indeed, a number of nonneoplastic pathologies can also appear as space-occupying lesions; these broadly include radiation necrosis, subacute infarct, demyelinating and inflammatory lesions, vasculopathies, and infectious lesions.,21,22 Tumefactive demyelinating lesions (TDLs), frequently resulting from multiple sclerosis (MS) or acute disseminated encephalomyelitis, are a well-known mimic of gliomas. Less common causes of TDLs include neuromyelitis optica spectrum disorders. TDLs are characterized as $>2-\mathrm{cm}$ lesions; additional MRI features include large white matter lesions, incomplete enhancement, irregular border, mass effect, involvement of gray matter, and variable T2-weighted signal intensity. ${ }^{23,24}$ TDLs as a result of MS can be differentiated from gliomas when the patient has a history of MS lesions or when more classic T2-hypersignal lesions in the periventricular areas are present. ${ }^{21,23}$ Multiple T2-hyperintense lesions, elevated relative cerebral blood volume, and glutamine-glutamate peaks on MR spectroscopy can also differentiate demyelinating lesions from gliomas. $^{25,26}$ The pattern of T2 hyperintensities in relation to vessels can also be useful when differentiating demyelinating disease from other CNS vasculopathies. A study by Maggi et al. demonstrated the high specificity of perivenular lesions for MS relative to other CNS 

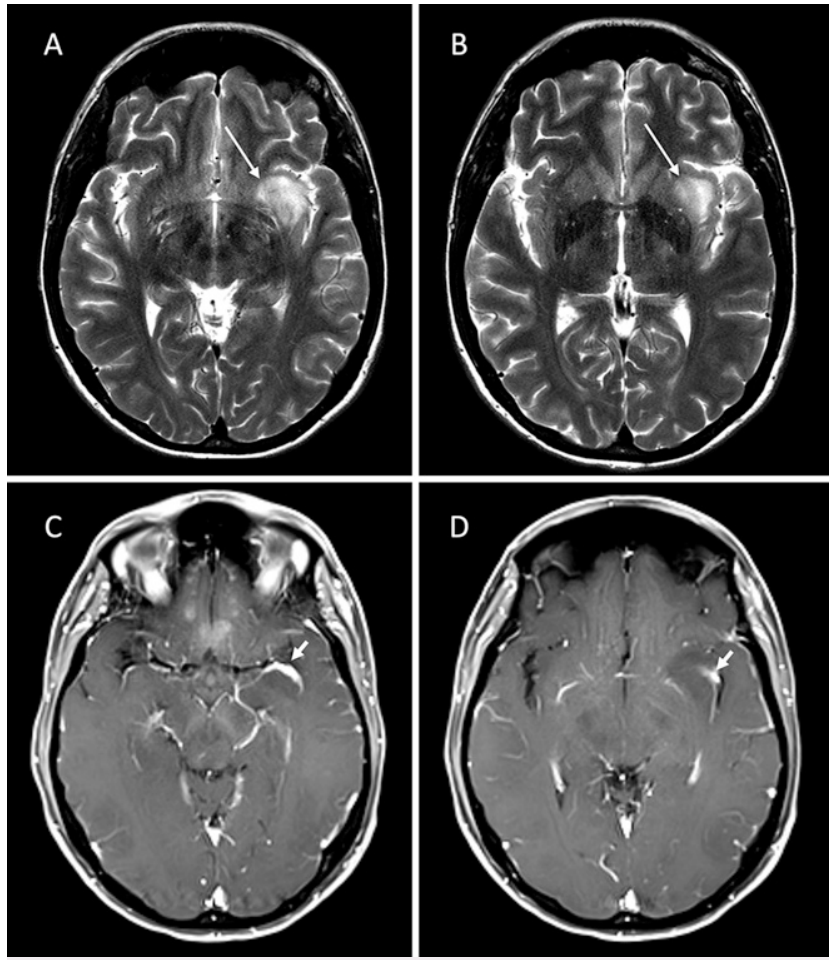

FIG. 3. Case 2 at initial presentation. A and B: Axial T2-weighted images show expansile mass lesion in the left insula (long arrows). $\mathbf{C}$ and D: Axial postcontrast T1-weighted images show curvilinear area of abnormal enhancement (short arrows) in the region of left MCA.

vasculopathies. ${ }^{27}$ This is particularly pertinent because the patients described in this report both had a periarterial lesion with contrast enhancement of the ipsilateral MCA, which would not be consistent with MS. Using noncontrast computed tomography (CT) in combination with MRI has also been shown to improve the ability of providers to differentiate between TDLs and glioma or primary central nervous system (CNS) lymphoma. In fact, Kim et al. demonstrated that the diagnostic accuracy of CT plus MRI was significantly higher than that of MRI $(97 \%$ versus $73.0 \% ; p<0.001$ ) when used to differentiate tumefactive lesions from true CNS neoplasms. ${ }^{23}$ Additional inflammatory processes that can present as a space-occupying CNS lesion include neurosarcoidosis, ${ }^{28}$ neuro-Behçet disease, ${ }^{29,30}$ autoimmune encephalitis, and TREX1-associated retinal vasculopathy with cerebral leukodystrophy. ${ }^{21,31}$

Similarly to the aforementioned demyelinating diseases, CNS vasculopathies can also present as space-occupying lesions. Indeed, up to $5 \%$ of cases of primary angiitis of the CNS (PACNS) can present as a brain tumor mimic. ${ }^{32-37}$ Vasculopathies can frequently demonstrate T2-hyperintense lesions with restricted diffusion on DWI in addition to leptomeningeal enhancement and underlying hemorrhage. $^{38,39}$ In addition, vasculopathies will most commonly present with headache and encephalopathy. ${ }^{40}$ However, Kim et al. reported a case of PACNS that presented as a T2-hyperintense mass lesion, with some contrast enhancement on T1-weighted postcontrast imaging, in a 20-year-old woman presenting with seizures, highlighting the ability for PACNS to present in a similar manner to a primary CNS neoplasm. ${ }^{33}$ A case series of four patients with PACNS-associated lesions reported by Lee et al. demonstrated variable MRI findings, including inconsistent findings across DWI and PWI, in each patient,
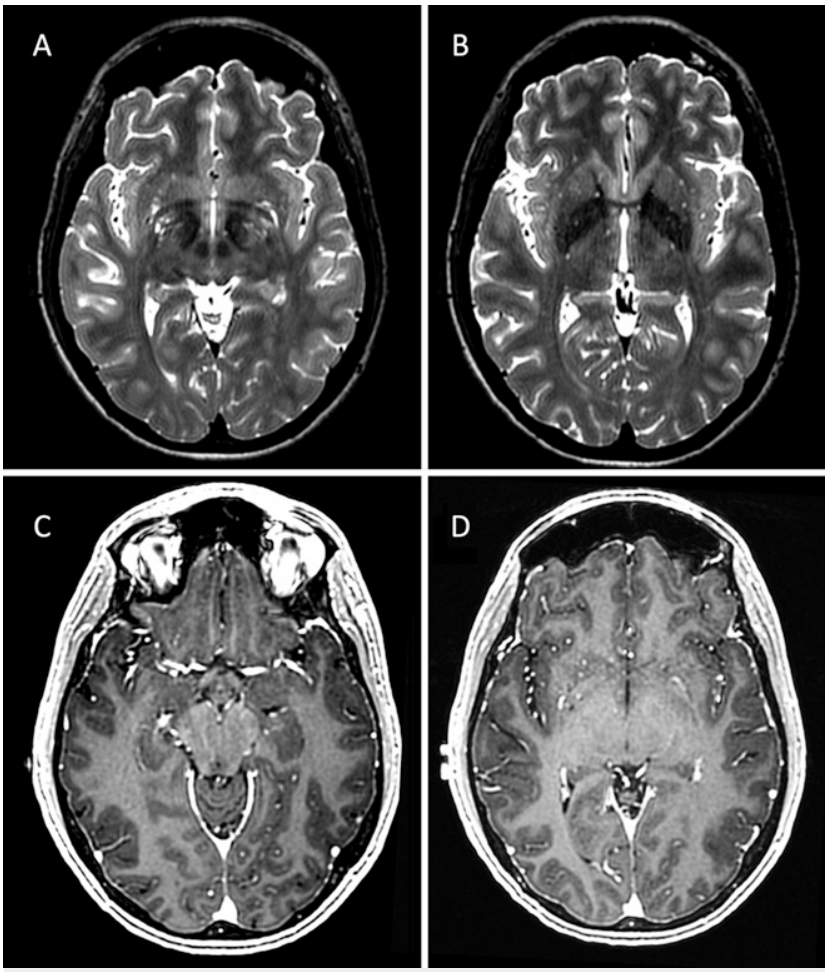

FIG. 4. Case 2 at preoperative MRI. A and B: Axial T2-weighted images show complete resolution of expansile mass lesion in the left insula. C and D: Axial postcontrast T1-weighted images show no residual enhancement in the region of left MCA.

demonstrating the heterogeneity of the disease and the potential for diagnostic difficulties. ${ }^{36}$ Similar mass lesions have been associated in other vasculopathies as well, including systemic lupus erythematosus, ${ }^{41}$ and granulomatosis with polyangiitis has also been reported in the literature. ${ }^{26,37}$ Lesions associated with vasculopathies will frequently resolve with steroid therapy, further supporting an underlying autoimmune or inflammatory process. ${ }^{36}$

The underlying causes of the two cases of spontaneously resolving insular mass lesions described in our report remain unclear, but insular glioma can be excluded. Although the lesions displayed MRI characteristics typically seen in LGGs, including an expansile, infiltrative mass with T2/FLAIR hyperintensity with hypointensity on T1weighted imaging, the presence of abnormal contrast enhancement around branches of the MCA and the resolution of the lesions without treatment differentiate them from LGGs (Table 1). Rather, these characteristics suggest a nonneoplastic cause of the imaging abnormality. The involvement of the MCA on T1 postcontrast imaging suggests a vasculopathy, such as those previously mentioned in this discussion. This is supported by the abnormal CSF studies in case 1 , including the presence of oligoclonal bands, which suggests an inflammatory process of uncertain etiology. Patients with findings similar to those described and with significant concern for vasculitis should undergo repeat MRI and be referred to neurology or rheumatology colleagues. Initial diagnostic steps include blood (e.g., erythrocyte sedimentation rate, C-reactive protein, complete blood count, complement studies, and ANA) and CSF (e.g., cell count, protein level, and presence of oligoclonal bands) work-up. If there is concern for large-vessel disease, diagnostic angiography can be 
TABLE 1. Characteristics of demyelinating diseases, CNS vasculopathies, and the present cases

\begin{tabular}{|c|c|c|c|c|}
\hline Characteristic & Multiple Sclerosis & Vasculopathy & Low-Grade Glioma & Present Cases \\
\hline Symptoms & $\begin{array}{c}\text { Variable, depending on location } \\
\text { of lesion } 44\end{array}$ & Headache, encephalopathy ${ }^{40}$ & $\begin{array}{l}\text { Seizure is most common. } \\
\text { Headache and focal neurological } \\
\text { deficit also seen. }{ }^{1,2}\end{array}$ & Headache \\
\hline Imaging & $\begin{array}{l}\text { Multiple T2-hyperintense white } \\
\text { matter lesions. Periventricular and/ } \\
\text { or perivenular lesions. Elevated } \\
\text { relative cerebral blood volume. } \\
\text { Glutamine-glutamine peaks on MR } \\
\text { spectroscopy. }\end{array}$ & $\begin{array}{c}\text { Can present with } \\
\text { T2-hyperintense lesions. } \\
\text { Restricted diffusion on DWI. } \\
\text { Leptomeningeal enhancement } \\
\text { and hemorrhagic lesions. Can be } \\
\text { variable and heterogeneous. }\end{array}$ & $\begin{array}{l}\text { T2-hyperintense mass lesion } \\
\text { following white matter distribution } \\
\text { without enhancement on T1 } \\
\text { postcontrast imaging. Can } \\
\text { demonstrate T2-FLAIR washout. } \\
\text { Increased choline peak and } \\
\text { decreased } N \text {-acetyl aspartate peak } \\
\text { on MR spectroscopy. }\end{array}$ & $\begin{array}{l}\text { T2-hyperintense lesion } \\
\text { in the insula. } \\
\text { Enhancement of the } \\
\text { ipsilateral MCA on T1 } \\
\text { postcontrast MRI. }\end{array}$ \\
\hline
\end{tabular}

considered to further assess the vasculopathy. Patients with a lesion consistent with incidental LGG should receive surgical resection, given the significant survival benefit associated with early surgery. ${ }^{42,43}$

\section{Lessons}

These cases highlight the need to maintain a broad differential diagnosis in the work-up of suspected insular LGG based on imaging and to obtain immediate preoperative imaging, ideally within 24-48 hours, for surgical planning, confirmation of the mass lesion, assessment of interval change, and high-quality MRI with DTI and fiducials. Avoiding corticosteroid treatment prior to the immediate preoperative imaging is also of importance so as not to confuse the diagnosis both on imaging and on pathologic evaluation. Perivascular MCA branch enhancement within the sylvian fissure may serve as a "warning sign" for pseudo-insular glioma syndrome.

\section{References}

1. Forst DA, Nahed BV, Loeffler JS, Batchelor TT. Low-grade gliomas. Oncologist. 2014;19(4):403-413.

2. Pouratian N, Asthagiri A, Jagannathan J, Shaffrey ME, Schiff D. Surgery insight: the role of surgery in the management of low-grade gliomas. Nat Clin Pract Neurol. 2007;3(11):628-639.

3. Duffau H, Capelle L. Preferential brain locations of low-grade gliomas. Cancer. 2004;100(12):2622-2626.

4. Parisot S, Darlix A, Baumann C, et al. A probabilistic atlas of diffuse WHO grade II glioma locations in the brain. PLoS One. 2016;11(1): e0144200.

5. Larsen J, Wharton SB, McKevitt F, et al. 'Low grade glioma': an update for radiologists. Br J Radiol. 2017;90(1070):20160600.

6. Brandão LA, Castillo M. Adult brain tumors: clinical applications of magnetic resonance spectroscopy. Neuroimaging Clin N Am. 2013;23(3):527-555.

7. Cha S. Update on brain tumor imaging. Curr Neurol Neurosci Rep. 2005;5(3):169-177.

8. Brown TJ, Bota DA, van Den Bent MJ, et al. Management of lowgrade glioma: a systematic review and meta-analysis. Neurooncol Pract. 2019;6(4):249-258.
9. Louis DN, Perry A, Wesseling P, et al. The 2021 WHO Classification of Tumors of the Central Nervous System: a summary. Neuro Oncol. 2021;23(8):1231-1251.

10. Kim MJ, Kim HS, Kim JH, Cho KG, Kim SY. Diagnostic accuracy and interobserver variability of pulsed arterial spin labeling for glioma grading. Acta Radiol. 2008;49(4):450-457.

11. Server A, Kulle B, Gadmar ØB, Josefsen R, Kumar T, Nakstad PH. Measurements of diagnostic examination performance using quantitative apparent diffusion coefficient and proton MR spectroscopic imaging in the preoperative evaluation of tumor grade in cerebral gliomas. Eur J Radiol. 2011;80(2):462-470.

12. Nguyen TB, Cron GO, Mercier JF, et al. Diagnostic accuracy of dynamic contrast-enhanced MR imaging using a phase-derived vascular input function in the preoperative grading of gliomas. AJNR Am J Neuroradiol. 2012;33(8):1539-1545.

13. Fouke SJ, Benzinger T, Gibson D, Ryken TC, Kalkanis SN, Olson JJ. The role of imaging in the management of adults with diffuse low grade glioma. J Neurooncol. 2015;125(3):457-479.

14. Delbeke D, Meyerowitz C, Lapidus RL, et al. Optimal cutoff levels of F-18 fluorodeoxyglucose uptake in the differentiation of low-grade from high-grade brain tumors with PET. Radiology. 1995;195(1): 47-52.

15. Calcagni ML, Galli G, Giordano A, et al. Dynamic O-(2-[18 F]fluoroethyl)-L-tyrosine (F-18 FET) PET for glioma grading: assessment of individual probability of malignancy. Clin Nucl Med. 2011;36(10): 841-847.

16. Shboul ZA, Chen J, Iftekharuddin MK. Prediction of molecular mutations in diffuse low-grade gliomas using MR imaging features. Sci Rep. 2020;10(1):3711.

17. van der Voort SR, Incekara F, Wijnenga MMJ, et al. Predicting the $1 p / 19 q$ codeletion status of presumed low-grade glioma with an externally validated machine learning algorithm. Clin Cancer Res. 2019;25(24):7455-7462.

18. Abdullah KG, Lubelski D, Nucifora PG, Brem S. Use of diffusion tensor imaging in glioma resection. Neurosurg Focus. 2013; 34(4):E1.

19. Wilden JA, Voorhies J, Mosier KM, O'Neill DP, Cohen-Gadol AA. Strategies to maximize resection of complex, or high surgical risk, low-grade gliomas. Neurosurg Focus. 2013;34(2):E5.

20. Wu JS, Zhou LF, Tang WJ, et al. Clinical evaluation and follow-up outcome of diffusion tensor imaging-based functional 
neuronavigation: a prospective, controlled study in patients with gliomas involving pyramidal tracts. Neurosurgery. 2007;61(5):935-949.

21. Omuro AM, Leite CC, Mokhtari K, Delattre JY. Pitfalls in the diagnosis of brain tumours. Lancet Neurol. 2006;5(11):937-948.

22. Guillevin R, Herpe G, Verdier M, Guillevin C. Low-grade gliomas: the challenges of imaging. Diagn Interv Imaging. 2014;95(10): 957-963.

23. Kim DS, Na DG, Kim KH, et al. Distinguishing tumefactive demyelinating lesions from glioma or central nervous system lymphoma: added value of unenhanced CT compared with conventional contrast-enhanced MR imaging. Radiology. 2009;251(2):467-475.

24. Algahtani $H$, Shirah B, Alassiri A. Tumefactive demyelinating lesions: a comprehensive review. Mult Scler Relat Disord. 2017:14:72-79.

25. Saini J, Chatterjee S, Thomas B, Kesavadas C. Conventional and advanced magnetic resonance imaging in tumefactive demyelination. Acta Radiol. 2011;52(10):1159-1168.

26. Rapalino O, Mullins ME. Intracranial infectious and inflammatory diseases presenting as neurosurgical pathologies. Neurosurgery. 2017;81(1):10-28.

27. Maggi $P$, Absinta M, Grammatico M, et al. Central vein sign differentiates multiple sclerosis from central nervous system inflammatory vasculopathies. Ann Neurol. 2018;83(2):283-294.

28. Bathla G, Singh AK, Policeni B, Agarwal A, Case B. Imaging of neurosarcoidosis: common, uncommon, and rare. Clin Radiol. 2016;71(1):96-106.

29. Bilge NŞY, Şaylõsoy S, Kaşifoglu T, Korkmaz C. Mass-like lesions as a rare form of neuro-Behçet's disease: a case report and review of the literature. Eur J Rheumatol. 2014;1(1):34-38.

30. Scully SE, Stebner FC, Yoest SM. Magnetic resonance spectroscopic findings in neuro-Behçet disease. Neurologist. 2004;10(6): 323-326.

31. Donahue JH, Patel SH, Fadul CE, Mukherjee S. Imaging mimics of brain tumors. Radiol Clin North Am. 2021;59(3):457-470.

32. Molloy ES, Hajj-Ali RA. Primary angiitis of the central nervous system. Curr Treat Options Neurol. 2007;9(3):169-175.

33. Kim SI, Kim SH, Cho HJ, et al. Mass-forming primary angiitis of central nervous system with Rosai-Dorfmann disease-like massive histiocytosis with emperipolesis. Pathol Int. 2015;65(8):420-425.

34. Kinsella JA, O'Brien W, Mullins GM, Brewer J, Whyte S. Primary angiitis of the central nervous system with diffuse cerebral mass effect and giant cells. J Clin Neurosci. 2010;17(5):674-676.

35. Muccio CF, Di Blasi A, Esposito G, Brunese L, D'Arco F, Caranci F. Perfusion and spectroscopy magnetic resonance imaging in a case of lymphocytic vasculitis mimicking brain tumor. Pol J Radiol. 2013;78(3):66-69.
36. Lee $\mathrm{Y}$, Kim JH, Kim E, et al. Tumor-mimicking primary angiitis of the central nervous system: initial and follow-up MR features. Neuroradiology. 2009;51(10):651-659.

37. Baaj AA, Vale FL, Carter JD, Rojiani AM. Granulomatosis with CNS involvement: a neuroimaging clinicopathologic correlation. J Neuroimaging. 2009;19(2):194-197.

38. Singh S, John S, Joseph TP, Soloman T. Primary angiitis of the central nervous system: MRI features and clinical presentation. Australas Radiol. 2003;47(2):127-134.

39. Campi A, Benndorf G, Filippi M, Reganati P, Martinelli V, Terreni MR. Primary angiitis of the central nervous system: serial MRI of brain and spinal cord. Neuroradiology. 2001;43(8):599-607.

40. Berlit P. Diagnosis and treatment of cerebral vasculitis. Ther $A d v$ Neurol Disord. 2010;3(1):29-42.

41. Huang $\mathrm{CH}$, Hung $\mathrm{CH}$, Chu YT, Hua YM. Tumor-like cerebral perivasculitis in a pediatric patient with systemic lupus erythematosus. Kaohsiung J Med Sci. 2008;24(4):218-222.

42. Ius $\mathrm{T}, \mathrm{Ng} \mathrm{S}$, Young JS, et al. The benefit of early surgery on overall survival in incidental low grade glioma patients: a multicenter study. Neuro Oncol. Published online September 8, 2021. doi: 10.1093/ neuonc/noab210.

43. Gogos AJ, Young JS, Pereira MP, et al. Surgical management of incidentally discovered low-grade gliomas. J Neurosurg. Published online October 2, 2020. doi: 10.3171/2020.6.JNS201296.

44. McGinley MP, Goldschmidt CH, Rae-Grant AD. Diagnosis and treatment of multiple sclerosis: a review. JAMA. 2021;325(8):765-779.

45. Hajj-Ali RA, Calabrese LH. Diagnosis and classification of central nervous system vasculitis. J Autoimmun. 2014;48-49:149-152.

\section{Disclosures}

The authors report no conflict of interest concerning the materials or methods used in this study or the findings specified in this paper.

\section{Author Contributions}

Conception and design: Haddad, Morshed, Berger. Acquisition of data: Haddad. Analysis and interpretation of data: Young, Cha, Berger. Drafting the article: Haddad, Young, Morshed. Critically revising the article: all authors. Reviewed submitted version of manuscript: all authors. Approved the final version of the manuscript on behalf of all authors: Haddad. Statistical analysis: Berger. Administrative/technical/ material support: Cha. Study supervision: Berger.

\section{Correspondence}

Alexander F. Haddad: University of California, San Francisco, San Francisco, CA. alexander.haddad@ucsf.edu. 